\title{
COUPLING AND DEPHASING IN JOSEPHSON CHARGE-PHASE QUBIT WITH RADIO FRE- QUENCY READOUT
}

\author{
Alexander B. Zorin *

\begin{abstract}
The Cooper pair box qubit of a two-junction-SQUID configuration enables the readout of the qubit states by probing the effective Josephson inductance of the SQUID. This is realized by coupling the qubit to a high-Q tank circuit which induces a small alternating supercurrent in the SQUID loop. The effect of a small (but finite) geometrical inductance of the loop on the eigenstates of the system is figured out. The effect of qubit dephasing due to quadratic coupling to the tank circuit is evaluated. It is shown that the rate of dephasing in the vicinity of the magic points is relatively low unless the Josephson junctions forming the qubit are rather dissimilar. In the vicinity of the avoided level-crossing point such dephasing is always significant.
\end{abstract}

\section{Introduction}

The readout device is a critical component of any potential quantum computing circuit. For the Josephson qubits there is a number of sensitive cryogenic devices available (SQUIDs, switching Josephson junctions, single electron transistors and traps, etc.) enabling the readout of the qubit state. However, operation of these devices is usually associated with a significant exchange of energy between detector and qubit, so in order to avoid fast decoherence the detector must be reliably decoupled from the qubit at the time of quantum manipulation. Recently, the class of Josephson qubit detectors based on the measurement of the reactive component of electrical signals related to nonlinear behavior of the Josephson inductance has been extensively studied [1, 2, 3, 4,. Due to specific coupling to the qubit variables

*A. B. Zorin, Physikalisch-Technische Bundesanstalt, 38116 Braunschweig, Germany. 


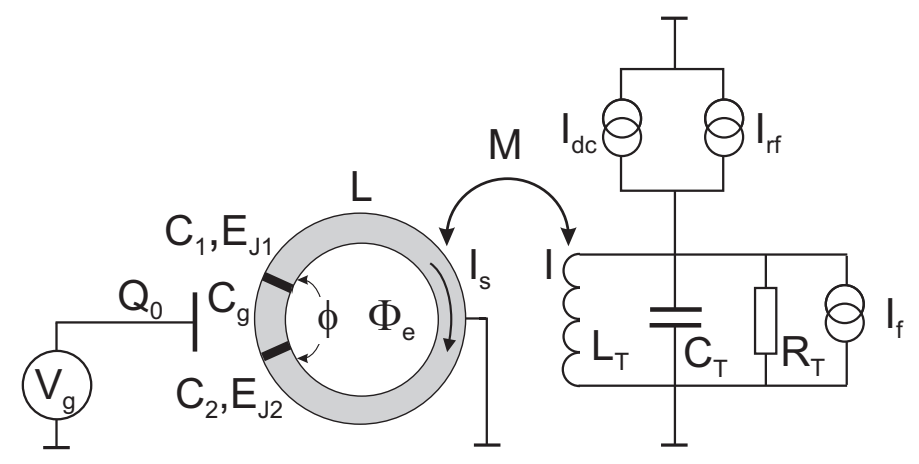

Figure 1: Electric circuit diagram of the charge-phase qubit. The core of the qubit is the superconducting ring of small inductance $L$ including two small tunnel junctions of capacitances $C_{1}$ and $C_{2}$ and Josephson coupling energies $E_{J 1}$ and $E_{J 2}$, respectively. The small island between the junctions is polarized by charge on small gate capacitance $C_{g}$. The ring is inductively (mutual inductance $M$ ) coupled to an rf-driven parallel tank circuit. Voltage across the tank circuit is read out by a (presumably cold) preamplifier which is not shown.

and non-dissipative characteristics of the Josephson supercurrent, these circuits can have a much weaker backaction and, therefore, cause lesser decoherence. Moreover, these circuits may possibly enable quantum nondemolition measurements of a Josephson qubit [5].

In this paper, we consider the charge-phase qubit [1] comprising a macroscopic superconducting ring including two small Josephson junctions with a small island in between (see Fig. 1), i.e., a Cooper pair box [6] of SQUID configuration. This setup is, in principle, similar to that of Quantronium [7, in which, however, a larger additional Josephson junction was inserted in the superconducting loop for the purpose of readout by switching this junction into a finite-voltage state. In our case, the ring of inductance $L$ is inductively coupled to the tank circuit that enables both phase control and readout of the qubit. The special convenience of this simple setup is that it can function even without uncoupling the tank circuit from the qubit. It has recently been shown that operation of this qubit in the so-called magic points made it possible to decouple (in linear approximation) both the diagonal and the off-diagonal components of the variables of the measuring system (environment) from the qubit [8]. Moreover, the resonance shape of the noise spectrum allowed significant reducing relaxation and, in principle, performing single shot measurements. Here we analyze the quadratic effect of this noise and show that dephasing caused by this effect in the vicinity of the magic operation points is small, provided the qubit is sufficiently symmetric. 


\section{Qubit parameters and the model}

For the vanishingly small value of inductance $L$ the behavior of the charge-phase qubit is primarily determined by the relationship between the energies associated with the island and the junctions. These are the Coulomb energy of the island, $E_{c}=e^{2} / 2 C_{\Sigma}$, and the Josephson coupling energies of the junctions, $E_{J 1, J 2}=$ $\hbar I_{c 1, c 2} / 2 e$, where $C_{\Sigma}=C_{1}+C_{2}+C_{g}$ is the island's total capacitance including the capacitances of the individual junctions $C_{1}$ and $C_{2}$ and the gate capacitance $C_{g} \ll C_{1,2} ; I_{c 1, c 2}$ are the nominal values of the Josephson critical currents. We assume that

$$
\left(\Phi_{0} / 2 \pi\right) I_{c 0}=E_{J 0} \equiv\left(E_{J 1}+E_{J 2}\right) / 2 \simeq E_{c},
$$

where $\Phi_{0}=h / 2 e$ is the flux quantum, $E_{J 0}$ and $I_{c 0}=\left(I_{c 1}+I_{c 2}\right) / 2$ are the average Josephson coupling energy and the average critical current, respectively. The coupling energies of individual junctions are almost similar, $E_{J 1} \approx E_{J 2}$, so the values of the dimensionless parameters $j_{1,2} \equiv E_{J 1, J 2} / 2 E_{J 0} \approx 0.5$, while $j_{1}+j_{2}=1$. The junction capacitances are also assumed to be almost similar, $C_{1} \approx C_{2}$, and the dimensionless parameters $\kappa_{1,2} \equiv C_{2,1} / C_{\Sigma} \approx 0.5$, while $\kappa_{1}+\kappa_{2}=1$. The qubit island is capacitively coupled to the gate voltage source $V_{g}$ which controls the polarization charge $Q_{0}=C_{g} V_{g}$. The inductively coupled coil $L_{T}$ carrying current $I$ induces the external magnetic flux $\Phi_{e} \equiv\left(\Phi_{0} / 2 \pi\right) \phi_{e}=-M I$ applied to the qubit loop and produces the sum phase bias $\phi \equiv \varphi_{1}+\varphi_{2}=\phi_{e}$. The core Hamiltonian includes the charging (kinetic) energy and Josephson (periodic potential) term,

$$
H_{0}=4 E_{c}\left(n-Q_{0} / 2 e\right)^{2}-E_{J}(\phi) \cos \chi,
$$

where the operator $n$ of the number of excess Cooper pairs on the island is

$$
n=-i \frac{\partial}{\partial \varphi}=-i \frac{\partial}{\partial \chi}, \quad[n, \varphi]=[n, \chi]=i .
$$

The operators of the island phases are $\varphi=\left(\varphi_{1}-\varphi_{2}\right) / 2$ and $\chi=\varphi+\gamma(\phi)$, where the angle deviation due to asymmetry of the junctions $\gamma=\arctan \left[\left(j_{1}-j_{2}\right) \tan (\phi / 2)\right]$. The amplitude of the effective Josephson coupling energy of two junctions is equal to

$$
E_{J}(\phi)=\left(E_{J 1}^{2}+E_{J 2}^{2}+2 E_{J 1} E_{J 2} \cos \phi\right)^{1 / 2} .
$$

The two lowest eigenvalues of energy, $E_{0}\left(Q_{0}, \phi\right)$ (ground state) and $E_{1}\left(Q_{0}, \phi\right)$ (first excited state), with corresponding eigenstates $|0\rangle$ and $|1\rangle$, form the basis suitable for qubit operation. In this basis the Hamiltonian Eq. (2) takes the diagonal form

$$
H_{0}=-(\epsilon / 2) \sigma_{z},
$$

where energy $\epsilon\left(Q_{0}, \phi\right) \equiv \hbar \Omega=E_{1}-E_{0}$ and $\sigma_{z}$ is the Pauli matrix. The energy landscape is $2 e$ periodic in $Q_{0}$ and $2 \pi$ periodic in $\phi$. The plots can be found elsewhere (see, for example, Refs. [7] and [8]). The reverse Josephson inductance of 
the qubit in the ground (excited) state $L_{0,1}^{-1}$ is determined by the local curvature of the corresponding energy surface,

$$
L_{0,1}^{-1}\left(Q_{0}, \phi\right)=\left(\frac{2 \pi}{\Phi_{0}}\right)^{2} \frac{\partial^{2} E_{0,1}\left(Q_{0}, \phi\right)}{\partial \phi^{2}} .
$$

Depending on the value of the ratio $E_{J 0} / E_{c}$ and the relationship between the Josephson energies of the junctions, $j_{1} / j_{2}$, the inductance $L_{0,1}$ can take either positive or negative values in different points on the $Q_{0}-\phi$ plane. In the so-called magic points (extremum or saddle points), i.e., $A\left(Q_{0}=0, \phi=\pi\right), B\left(Q_{0}=0, \phi=\right.$ $0)$ and $C\left(Q_{0}=e, \phi=0\right)$, the absolute values of $L_{0,1}^{-1}$ achieve local maxima, while in the avoided level-crossing point $D\left(Q_{0}=e, \phi=\pi\right)$ its value is the largest 8 . Specifically, in point $D$, where effective coupling is small, $E_{J}(\pi)=\left|E_{J 1}-E_{J 2}\right| \ll$ $E_{c}$, these values for two states are equal to 9

$$
L_{0,1}^{-1}(D)=\mp \frac{2 \pi}{\Phi_{0}} \frac{j_{1} j_{2}}{\left|j_{1}-j_{2}\right|} I_{c 0} .
$$

The drive frequency $\omega_{\text {rf }}$ (close to the resonance frequency of the tank circuit $\left.\omega_{T}=\left(L_{T} C_{T}\right)^{1 / 2} \ll k_{B} T / \hbar\right)$ is much lower than the qubit frequency $\Omega$, so the induced classical oscillations of phase $\phi$ are adiabatic. Due to coupling to the ring, the effective inductance seen by the tank circuit is 10

$$
L_{\mathrm{eff}}^{(0,1)}=L_{T}-\frac{M^{2}}{L+L_{0,1}} \approx L_{T}-\frac{M^{2}}{L_{0,1}} .
$$

As a result, the resonance frequencies take the distinct values for the ground and excited states, $\omega_{0,1}=\left(L_{\text {eff }}^{(0,1)} C_{T}\right)^{-1 / 2}$, and this property is used for the radio frequency readout of the qubit [1].

\section{Effect of finite inductance of the ring}

The problem of the inductance effects in the persistent current flux qubits had been addressed by Crankshaw and Orlando in Ref. [11] where the corrections to the energy level values of these qubits were found. Recently, Maassen van den Brink had analyzed the finite inductance effect in the three-Josephson-junction jubits considering the self-flux as a "fast variable" 12. He had shown that for small inductances this effect is merely reduced to renormalization of the individual Josephson coupling energies entering the system Hamiltonian.

In the case of the charge-phase qubit, the sum phase $\phi$ is also no longer a good variable. Instead, its combination with the normalized circulating current (which is a quantum-mechanical operator) is controlled by the external flux $\Phi_{e}$,

$$
\phi+\frac{2 \pi}{\Phi_{0}} L I_{s}(\phi, \chi)=\phi_{e} .
$$


The operator of the circulating current can be presented as $\underline{8}$

$$
I_{s}=I_{\|}(\phi) \cos \chi+I_{\perp}(\phi) \sin \chi
$$

In the given basis, the operator $\cos \chi$ is diagonal and $\sin \chi$ is off-diagonal, so the amplitudes of the longitudinal $\left(I_{\|}\right)$and transversal $\left(I_{\perp}\right)$ components of current $I_{s}$ are

$$
\begin{gathered}
I_{\|}=\frac{8 \pi}{\Phi_{0}} j_{1} j_{2} \sin \phi \frac{E_{J 0}^{2}}{E_{J}(\phi)}, \\
I_{\perp}=\frac{8 \pi}{\Phi_{0}}\left[\left(j_{1}-j_{2}\right)\left(\kappa_{1} j_{1}+\kappa_{2} j_{2}\right)+2 j_{1} j_{2}\left(\kappa_{1}-\kappa_{2}\right) \cos ^{2} \frac{\phi}{2}\right] \frac{E_{J 0}^{2}}{E_{J}(\phi)} .
\end{gathered}
$$

Note that the operator $I_{s}$ is diagonal for arbitrary values of parameters $Q_{0}$ and $\phi$ only if the junctions are identical, i.e., $j_{1}=j_{2}$ and $\kappa_{1}=\kappa_{2}$, so $I_{\perp}=0$.

The additional magnetic energy term in the system Hamiltonian is

$$
H_{m}=\left(\Phi_{0} / 2 \pi\right)^{2}\left(\phi-\phi_{e}\right)^{2} / 2 L=L I_{s}^{2}(\phi, \chi) / 2 .
$$

The kinetic energy term associated with the charging of the chain of the qubit junctions connected in series, $H_{\tilde{c}}=\left(\Phi_{0} / 2 \pi\right)^{2} \tilde{C} \dot{\phi}^{2} / 2$, where $\tilde{C}=C_{\text {qubit }}+C_{\text {stray }}$. Although the capacitance between the ends of the SQUID loop $C_{\text {stray }}$ depends on the sample layout, it clearly dominates over the first term, $C_{\text {qubit }}=C_{1} C_{2} / C_{\Sigma}$ which is typically about $1 \mathrm{fF}[13]$. The resonance frequency $\omega_{L}=(L \tilde{C})^{1 / 2}$ of the oscillator formed by $L$ and $\tilde{C}$ is therefore relatively low. Assuming $\hbar \omega_{L} \sim k_{B} T$, but still $\omega_{L} \gg \omega_{\mathrm{rf}}$ we recover classical behavior of $\phi$. In this case it is sufficient to take into account only the magnetic term associated with the circulating current given by Eq. (13).

The phase variable $\phi$ is a single-valued function of $\phi_{e}$ for arbitrary values of $Q_{0}$, provided $L$ is sufficiently small, viz.,

$$
L\left|L_{0,1}^{-1}\right| \leq L\left|L_{0,1}^{-1}(D)\right|=\frac{j_{1} j_{2}}{\left|j_{1}-j_{2}\right|} \beta_{L}<1,
$$

where the screening parameter is

$$
\beta_{L}=\frac{2 \pi}{\Phi_{0}} L I_{c 0}
$$

One can see that for an almost symmetric qubit $\left(j_{1} \approx j_{2}\right)$ the condition of smallness of inductance $L$, i.e. $\beta_{L}<4\left|j_{1}-j_{2}\right|$, is more stringent than that for ordinary rf SQUIDs, i.e. $\beta_{L}<1$ [14].

To derive the dependence $\phi\left(\phi_{e}\right)$, one can solve Eq. (9) by iteration. The first step gives the expression

$$
\phi \approx \phi_{e}-\left(2 \pi / \Phi_{0}\right) L I_{s}\left(\phi_{e}, \chi\right)
$$


A. B. Zorin

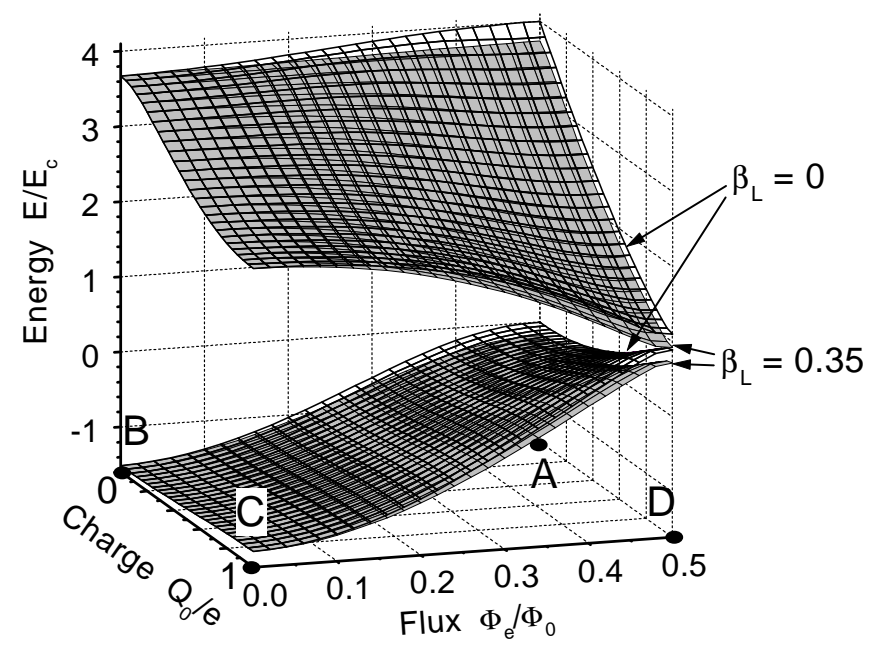

Figure 2: Energies $E_{0}$ and $E_{1}$ calculated for the zero (wire frames) and nonzero (surface plots) values of screening parameter $\beta_{L}$ for the case of mean Josephson coupling $E_{J 0} \equiv$ $\frac{1}{2}\left(E_{J 1}+E_{J 2}\right)=2 E_{c}$ and the asymmetry parameter $a \equiv j_{1}-j_{2}=\left(E_{J 1}-E_{J 2}\right) /\left(E_{J 1}+\right.$ $\left.E_{J 2}\right)=0.1$. Black dots mark on the $Q_{0}-\Phi_{e}$ plane the locations of the magic points $A, B$, and $C$ and the avoided level-crossing point $D$.

Then the corresponding one-dimensional Schrödinger equation,

$$
\left\{-4 E_{c}\left(\frac{\partial}{\partial \chi}-i \frac{Q_{0}}{2 e}\right)^{2}-E_{J}\left(\phi_{e}\right) \cos \chi-\frac{L}{4}\left[I_{\|}\left(\phi_{e}\right) \cos \chi+I_{\perp}\left(\phi_{e}\right) \sin \chi\right]^{2}\right\} \psi=E \psi,
$$

is again of the type describing a quantum particle moving in a periodic potential. In the case of $L=0$, this potential is harmonic and the equation is of the Mathieu type [15]. In the case of nonzero $L$, the potential energy includes a (small) second harmonic admixture. The eigenvalues and eigenfunctions of this equation are found by numerical methods.

Figure 2 shows the two lowest eigenvalues of energy (the qubit levels) computed from the Schrödinger equation Eq. (17). For the given parameters of the qubit and not very small value of $\beta_{L}$, the subsequent terms of iteration give substantially smaller correction. One can see that similar to the conclusion of Ref. 11], the first order effect of inductance reduces to the lowering of both energy surfaces, $E_{0}$ and $E_{1}$. The correction to the level spacing Eq. (5) is, however, very small: $\epsilon \rightarrow \epsilon+\Delta \epsilon_{L}, \Delta \epsilon_{L} \ll \epsilon$. As can be seen from Fig. 2, the effect of the deformation of the energy surfaces is most appreciable in the region of small effective Josephson coupling combined with charge degeneracy, e.g., in the vicinity of point $D$. Around the magic points $A$ and - especially - $B$ and $C$, which are most suitable for qubit operation, the change of local curvature of the surfaces is rather small. 


\section{Qubit dephasing}

The term in the system Hamiltonian which describes coupling of the qubit and the variable $\delta \Phi_{e}=M I_{f}$ associated with the external flux noise induced in the ring by the tank circuit is given by

$$
H_{\text {coupl }}=-I_{s} \delta \Phi_{e}
$$

or, in the qubit basis Eq. (5),

$$
H_{\text {coupl }}=-\frac{1}{2} U_{z} \sigma_{z}-\frac{1}{2} U_{y} \sigma_{y}=-\frac{1}{2}\left[\left(c_{11}-c_{00}\right) I_{\|}\left(\phi_{e}\right) \sigma_{z}+2 s_{01} I_{\perp}\left(\phi_{e}\right) \sigma_{y}\right] \delta \Phi_{e},
$$

where corresponding matrix elements are denoted by

$$
c_{00}=\langle 0|\cos \chi| 0\rangle, \quad c_{11}=\langle 1|\cos \chi| 1\rangle, \quad \text { and } \quad s_{01}=|\langle 0|\sin \chi| 1\rangle| .
$$

The remarkable property of the coupling described by Eq. (19) implies that the longitudinal term, $U_{z} \propto I_{\|}$, vanishes in the optimal points $A, B, C$ and $D$, i.e. where $\sin \phi_{e}=0$ (see Eq. (11)) [16. The transversal term, $U_{y} \propto I_{\perp}$, in these points is, however, nonzero, and its low-frequency $(\ll \Omega)$ noise component can dephase the qubit due to renormalization of the level spacing [17], $\epsilon \rightarrow\left(\epsilon^{2}+U_{y}^{2}\right)^{1 / 2} \approx \epsilon+U_{y}^{2} / 2 \epsilon$, yielding the diagonal term

$$
-\frac{1}{2} X \sigma_{z} \equiv-\frac{U_{y}^{2}}{2 \epsilon} \sigma_{z}=-\frac{2}{\epsilon} s_{01}^{2} I_{\perp}^{2}\left(\phi_{e}\right)\left(\delta \Phi_{e}\right)^{2} \sigma_{z}
$$

The problem of qubit dephasing due to quadratic longitudinal coupling to the environment was addressed by Makhlin and Shnirman in Ref. [17] where they focused on the experimentally relevant $1 / f$ and the Johnson-Nyquist noise power spectra. Here we extend this model to our case of a structured bath (low-frequency resonance tank circuit).

For white noise $I_{f}$ associated with losses in resistance $R_{T}$, the power spectrum density $S_{\Phi}(\omega)$ of variable $\delta \Phi_{e}$ is simply found from the network consideration. Neglecting small detuning of the tank due to coupling to the qubit ring $\left(\omega_{0} \approx \omega_{1} \approx\right.$ $\omega_{T}$ ) we arrive at the expression,

$$
S_{\Phi}(\omega)=M^{2} \frac{k_{B} T}{\pi R_{T}} \frac{\omega_{T}^{4}}{\left(\omega^{2}-\omega_{T}^{2}\right)^{2}+\omega^{2} \omega_{T}^{2} Q^{-2}}, \quad-\infty<\omega<\infty,
$$

where $Q=\omega_{T} C_{T} R_{T}=R_{T} / \omega_{T} L_{T}$ is the quality factor. Assuming that the noise is Gaussian we find the power spectrum density of fluctuations of the variable $\left(\delta \Phi_{e}\right)^{2}$ (see, for example, Ref. [18]),

$$
S_{\Phi^{2}}(\omega)=2 \int_{-\infty}^{+\infty} d \omega^{\prime} S_{\Phi}\left(\omega^{\prime}\right) S_{\Phi}\left(\omega^{\prime}-\omega\right)
$$

Taking this integral at $\omega \rightarrow 0$ yields the value

$$
S_{\Phi^{2}}(0)=\frac{1}{\pi} M^{4} Q^{3} \omega_{T}\left(\frac{k_{B} T}{R_{T}}\right)^{2}
$$


which determines the power spectrum density $S_{X}(0)$ of variable $X$,

$$
S_{X}(0)=\frac{2}{\epsilon^{2}} s_{01}^{4} I_{\perp}^{4} S_{\Phi^{2}}(0),
$$

and the rate of "pure" dephasing in the Bloch-Redfield approximation [19], $\Gamma_{\varphi}=$ $S_{X}(0) / \hbar^{2}$. Finally, the qubit quality factor $Q_{\varphi} \equiv \pi \Omega / \Gamma_{\varphi}$ can be presented as

$$
Q_{\varphi}=\frac{\pi}{2}\left(\frac{I_{c 0}}{s_{01} I_{\perp}}\right)^{4}\left(k^{2} Q \beta_{L}\right)^{-2} \frac{\epsilon^{3} \hbar \omega_{T}}{\left(E_{J 0} k_{B} T\right)^{2}} Q
$$

where $k=M /\left(L L_{T}\right)^{1 / 2}$ is the dimensionless coupling coefficient.

To evaluate $Q_{\varphi}$ for typical parameters of the qubit (leading for the operation points $A, B$ and $C$ to the value $\left.\left(\epsilon / E_{J 0}\right)^{3} \sim 10\right)$ let us assume that the quality factor of the tank circuit is $Q \sim 300$ while the product $k^{2} Q \beta_{L} \sim 10$ which ensures sufficient resolution in determining the resonance frequencies $\omega_{0,1}$. Taking the value of ratio $E_{J 0} \hbar \omega_{T} /\left(k_{B} T\right)^{2} \sim 0.1$ (with $E_{J 0}=100 \mu \mathrm{eV}, \omega_{T} / 2 \pi=100 \mathrm{MHz}$ and $T=1 \mathrm{~K}$ ) we get an estimate: $Q_{\varphi} \sim 10^{2}\left(I_{c 0} / s_{01} I_{\perp}\right)^{4}$.

In the optimal points $A$ and $D$, the amplitude $I_{\perp}$ (see Eq. (12)) is equal to

$$
\left(I_{\perp}\right)_{A, D}=2\left(\kappa_{1} j_{1}+\kappa_{2} j_{2}\right) I_{c 0} \approx I_{c 0},
$$

while in points $B$ and $C$

$$
\begin{aligned}
\left(I_{\perp}\right)_{B, C} \quad & =2\left[\left(j_{1}-j_{2}\right)\left(\kappa_{1} j_{1}+\kappa_{2} j_{2}\right)+2 j_{1} j_{2}\left(\kappa_{1}-\kappa_{2}\right)\right] I_{c 0} \\
& \approx\left(j_{1}-j_{2}+\kappa_{1}-\kappa_{2}\right) I_{c 0} .
\end{aligned}
$$

The values of the matrix element $s_{01}$ in points $A$ and $B$ are equal to 8

$$
\left(s_{01}\right)_{A, B} \approx \frac{\left|j_{1} \mp j_{2}\right|}{8 \sqrt{2}} \frac{E_{J 0}}{E_{c}},
$$

while in points $C$ and $D$

$$
\left(s_{01}\right)_{C, D} \approx 0.5 .
$$

Summarizing, the order-of-magnitude estimates for parameter $Q_{\varphi}$ in different operation points are

$$
\begin{aligned}
\left(Q_{\varphi}\right)_{A} & \sim 10^{4}\left(j_{1}-j_{2}\right)^{-4}, \\
\left(Q_{\varphi}\right)_{B} & \sim 10^{4}\left(j_{1}-j_{2}+\kappa_{1}-\kappa_{2}\right)^{-4}, \\
\left(Q_{\varphi}\right)_{C} & \sim 10\left(j_{1}-j_{2}+\kappa_{1}-\kappa_{2}\right)^{-4}, \\
\left(Q_{\varphi}\right)_{D} & \sim 10 .
\end{aligned}
$$

For the Josephson junctions with a reasonably high symmetry in their parameters, say $\pm 10 \%$ (e.g., $j_{1}-j_{2}=0.1$ ), the expected value of $Q_{\varphi}$ in points $A, B$ and $C$ greatly exceeds the level $10^{5}$ which should be sufficient for realization of active schemes for compensation of decoherence. In contrast to these figures, the value achieved in the avoided level-crossing point $D$ is relatively low. 


\section{Conclusion}

We have shown that qubit dephasing due to nonlinear coupling in the vicinity of magic operation points is relatively weak if asymmetry of the junction parameters is sufficiently small and the effective temperature of the tank circuit sufficiently low. Since this temperature is associated with the backaction noise of a preamplifier, its noise figure should be sufficiently low, as, for example, in the case of a cold semiconductor-based amplifier or a dc-SQUID-based amplifier 20. Further improvement (if necessary) of the charge-phase qubit with radio frequency readout can be achieved by using a stage ensuring variable coupling between the ring and the tank circuit. This could be, for example, a flux transformer with a variable transfer function [21].

In conclusion, sufficiently symmetric parameters of the junctions make it possible to effectively protect the charge-phase qubit against dephasing caused by its coupling to the radio-frequency readout circuit. The dominating mechanisms which may still cause appreciable dephasing in this kind of qubit are most likely related to intrinsic characteristics of the Josephson junctions (see, e.g., Refs. 22, 23]).

\section{Acknowledgment}

I would like to thank Alec Maassen van den Brink, Apostol Vourdas and Denis Vion for helpful comments. This work was partially supported by the EU through the SQUBIT-2 project.

\section{References}

[1] A. B. Zorin, "Cooper-pair qubit and Cooper-pair electrometer in one device," Physica C 368, 284-288 (2002).

[2] E. Il'ichev, A.Yu. Smirnov, M. Grajcar, A. Izmalkov, D. Born, N. Oukhanski, Th. Wagner, W. Krech, H.-G. Meyer, and A.M. Zagoskin, "Radio-frequency method for investigation of quantum properties of superconducting structures," cond-mat/0402559 and references therein.

[3] A. Lupaşcu, C. J. M. Verwijs, R. N. Schouten, C. J. P. M. Harmans, and J. E. Mooij, "Nondestructive readout for a superconducting flux qubit," cond-mat/0311510

[4] I. Siddiqi, R. Vijay, F. Pierre, C.M. Wilson, M. Metcalfe, C. Rigetti, L. Frunzio, and M.H. Devoret, "An RF-driven Josephson bifurcation amplifier for quantum measurements," cond-mat/0312623

[5] D. V. Averin, "Quantum nondemolition measurements of a qubit," Phys. Rev. Lett. 88, 207901 (2002).

[6] V. Bouchiat, D. Vion, P. Joyez, D. Esteve and M. H. Devoret, "Quantum coherence with a single Cooper pair," Phys. Scr. T76, 165-170 (1998).

[7] D. Vion, A. Aassime, A. Cottet, P. Joyez, H. Pothier, C. Urbina, D. Esteve and M. H. Devoret, "Manipulating the quantum state of an electrical current," Science 296, 886-889 (2002). 
[8] A. B. Zorin, "Josephson charge-phase qubit with radio frequency readout: coupling and decoherence," Zh. Éksp. Teor. Fiz. 125(6), 1423-1435 (2004) [JETP 98(6), 1250-1261 (2004)].

[9] W. Krech, M. Grajcar, D. Born, I. Zhilyaev, Th. Wagner, E. Il'ichev, and Ya. Greenberg, "Dynamic features of a charge qubit closed by a superconducting inductive ring," Phys. Lett. A 303, 352-357 (2002).

[10] R. Rifkin and B. S. Deaver, Jr., "Charge-phase relation and phase-dependent conductance of superconducting point contacts from rf impedance measurements," Phys. Rev. B 13(9), 3894-3901 (1976).

[11] D.S. Crankshaw and T.P. Orlando, "Inductance effects in the persistent current qubit," IEEE Appl. Supercond. 11(1), 1006-1009 (2001).

[12] A. Maassen van den Brink, "Hamiltonian for coupled qubits," cond-mat/0310425

[13] In the rf-SQUID circuits comprising Josephson weak-links instead of tunnel junctions a sufficiently small value of effective capacitance $\tilde{C}$ and, hence high $\omega_{L}$ may cause quantum behavior of phase $\phi$; see, for example, R. Whiteman, V. Schöllmann, M. Everitt, T. D. Clark, R. J. Prance, H. Prance, J. Diggins, G. Buckling, and J.F. Ralf, "Adiabatic modulation of a superconducting quantum interference device (SQUID) ring by an electromagnetic field," J. Phys.: Condens. Matter 10, 9951-9968 (1998).

[14] P. K. Hansma, Superconducting single-junction interferometers with small critical currents, J. Appl. Phys. 44(9), 4191-4194 (1973).

[15] Handbook of Mathematical Functions, edited by M. Abramowitz and I. A. Stegun (U.S. GPO, Washingtin, D.C., 1972), Chapter 20.

[16] In particular, quantum manipulation at a remarkably low dephasing rate of the Quantronium qubit was performed in operation point $C$ [7].

[17] Yu. Makhlin and A. Shnirman, "Dephasing of solid-state qubits at optimal points," cond-mat/0308297

[18] S. A. Akhmanov, Yu.E. Dyakov and A.S. Chirkin, Vvedenie v Statisticheskuyu Radiofiziku i Optiku (Introduction to Statistical Radiophysics and Optics), (Nauka, Moscow, 1981), Chapter 5 - in Russian.

[19] A. Shnirman, Yu. Makhlin and G. Schön, Noise and decoherence in quantum twolevel systems, Phys. Scr. T102, 147-154 (2002).

[20] M. Mück, J. B. Kycia and J. Clarke, "Superconducting quantum interference device as a near-quantum-limited amplifier at $0.5 \mathrm{GHz}$," Appl. Phys. Lett. 78(7), 967969 (2001).

[21] C. Cosmelli, M. G. Castellano, F. Chiarello, R. Leoni, D. Simeone, G. Torrioli, and P. Carelli, "Controllable flux coupling for the integration of flux qubits," cond-mat/0403690

[22] E. Paladino, L. Faoro, G. Falci, and R. Fazio, "Decoherence and $1 / f$ noise in Josephson qubits," Phys. Rev. Lett. 88, 228304 (2002).

[23] D. J. Van Harlingen, T. L. Robertson, B. L. T. Plourde, P. A. Reichardt, T. A. Crane, and J. Clarke, "Decoherence in Josephson-junction qubits due to critical current fluctuations," cond-mat/0404307 